\title{
Tooling up for microbiota research
}

Two new papers published in Cell describe powerful new genetic tools to characterize and manipulate the gut microbiota, opening up previously sealed avenues of research.

The explosion of interest in the contribution of the gut microbiota to host health and disease in the past decade has been accompanied and driven by ongoing refinements of investigational techniques. As the focus of research shifts to how microbiota promote healthy or diseased states, new tools are required to define the function of specific gut microorganisms and microbial genes.

In these new studies, Whitaker et al. and Lim et al. sought to develop genetic techniques to modify Bacteroides, which represents the most abundant genus of bacteria in the human gut. "Relatively few genetic tools are available for manipulating these bacteria, compared to more common lab strains like Escherichia coli," explains Weston Whitaker.

By adapting a Bacteroides integration plasmid to be compatible with the Golden Gate cloning approach, Whitaker and colleagues developed a rapid, accurate and efficient means of introducing fluorescent reporter genes to Bacteroides. To ensure that these genes were expressed at levels enabling visualization via fluorescence microscopy, historically challenging in these bacteria, stronger promoter and ribosomal binding site sequences were designed and validated. Not only did these new regulatory regions markedly increase synthesis of the fluorescent reporter, but this heterologous expression did not impair bacterial fitness in mice. In six Bacteroides spp. engineered to express different fluorescent reporters, Whitaker and colleagues could differentiate each species at a single-cell level in mouse colon sections.

In their study, Bentley Lim and colleagues focused on designing an inducer system to enable functional interrogation of Bacteroides genes, independent of endogenous gene regulation machinery. "We started by identifying consensus promoter elements conserved across diverse Bacteroides genomes," describes author Andrew Goodman. "We then used sequence conservation to predict promoter regions that would be permissive for engineering regulatory elements."

By designing an inducer system for use in Bacteroides responsive to the tetracycline analogue anhydrotetracycline, the investigators avoided issues with previous inducer systems in this genus that activated aberrantly in response to polysaccharides native to the host or present in the diet. Moreover, the inducer could be administered in drinking water, with different concentrations of anhydrotetracycline

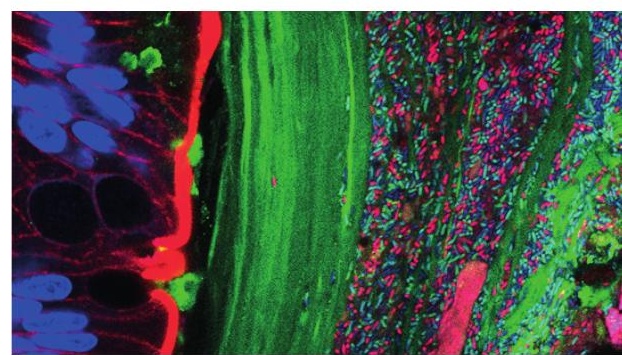

Image courtesy of J. Sonnenburg.

inducing dose-responsive changes in target gene expression.

Sialic acid, liberated from the gut mucosa by the action of sialidases expressed by some commensal bacteria, is an important nutrient source for pathogenic microorganisms including Clostridium difficile. "By colonizing mice with Bacteroides spp. whose sialidase expression was controlled by the synthetic inducer, we were able to measure the relationship between commensal sialidase activity and modification of the host environment," Goodman explains. Notably, this approach enabled the investigators to determine that sialic acid levels in the gut are limited by substrate availability, and not by commensal sialidase activity.

\section{Hugh Thomas}

ORIGINAL ARTICLES Whitaker, W. R. et al. Tunable expression tools enable single-cell strain distinction in the gut microbiome. Cell 169, 538-546.e12 (2017) | Lim, B. et al. Engineered regulatory systems modulate gene expression of human commensals in the gut. Cell 169, 547-558.e15 (2017) 